\title{
KRAS Mutation Analysis Was Performed
}

National Cancer Institute

\section{Source}

National Cancer Institute. KRAS Mutation Analysis Was Performed. NCI Thesaurus. Code C160397.

An indication that KRAS mutation analysis was performed during the study. 